\title{
Five-year outcomes after revascularization of superficial femoral artery occlusion using Ocelot catheter
}

\author{
Przemysław Nowakowski ${ }^{1,2}$, Piotr Buszman ${ }^{3,4}$, Adam Janas ${ }^{4}$, Stefan Kiesz ${ }^{5}$, Pawel Buszman ${ }^{4}$ \\ ${ }^{1}$ Department of Vascular Surgery, American Heart of Poland, Chrzanow, Poland \\ ${ }^{2}$ Katowice University of Technology, Katowice, Poland \\ ${ }^{3}$ Andrzej Frycz Modrzewski Krakow University, Krakow, Poland \\ ${ }^{4}$ Center for Cardiovascular Research and Development, Katowice, Poland \\ ${ }^{5}$ San Antonio Endovascular and Heart Institute, San Antonio, USA
}

Adv Interv Cardiol 2019; 15, 4 (58): 472-476

DOI: https://doi.org/10.5114/aic.2019.90222

\begin{abstract}
A bstract
Introduction: The population of patients with lower limb atherosclerosis includes a considerable proportion of individuals with long superficial femoral artery (SFA) lesions. Chronic total occlusions (CTOs) represent the "last frontier" of percutaneous interventions. While open strategies are considered earlier as standard management for these lesions, the results of a number of trials indicate that endovascular management might become an effective alternative to surgery.

Material and methods: This paper presents 5-year outcomes of a first-in-man (FIM) study (before CE mark) and the registry of OCT Guided Ocelot Catheter (Avinger) for chronic total occlusions of the superficial femoral artery. The study group comprised 10 patients with Rutherford 3 lower limb ischemia including nine men and one woman.

Results: The efficacy of the primary intervention was $90 \%$. Angiography performed at 6 months of the procedure, according to the study protocol, revealed 3 and 1 cases of restenosis and reocclusion, respectively, repaired using PTA and open common and deep femoral artery patch plasty. Doppler ultrasound performed at 1, 2 and 5 years after the primary intervention did not reveal significant target vessel restenosis. The primary and primary-assisted patency was $89 \%$. During a 5 -year follow-up, four peripheral percutaneous interventions and one femoropopliteal bypass surgery were performed in non-target limbs. There were no cardiovascular deaths, myocardial infarction or stroke and no amputation was required.

Conclusions: This is a first-in-man study reporting long-term follow-up after SFA CTO revascularization using the Ocelot catheter. The catheter proved to have a satisfactory safety profile and a high proportion of CTO crossings. A 5-year follow-up revealed high primary and primary-assisted patency rates.
\end{abstract}

Key words: revascularization, superficial femoral artery occlusion, optical coherence tomography.

Su m m a ry

The limitations of this FIM study are its non-randomized nature and small sample size. A distinct advantage was that follow-up tools included not only Doppler ultrasound but also angiography, which was in accordance with the study protocol. In conclusion, this is a first-in-man study reporting long-term follow-up after superficial femoral artery (SFA) chronic total occlusion (СTO) revascularization using the Ocelot catheter. The catheter proved to have a satisfactory safety profile and a high proportion of CTO crossings. A 5-year follow-up revealed high primary and primary-assisted patency rates.

\section{Introduction}

Chronic total occlusions (CTO) of the superficial femoral artery (SFA) are historically recommended by TASC II as a subgroup where surgical intervention is preferred over endovascular revascularization [1]; according to literature reports approximately $40 \%$ of patients with symptomatic peripheral arterial disease have CTO of SFA [1]. Therefore, long SFA occlusion is frequently referred to as an "Achilles heel" and represents the "last frontier" of lower extremity percutaneous revascularizations [2-4].

\section{Corresponding author:}

Przemysław Nowakowski MD PhD, Department of Vascular Surgery, American Heart of Poland, Chrzanów, Poland, phone: +48 326258120 , e-mail: nowakowski.mcsn@gmail.com

Received: 9.03.2019, accepted: 14.07.2019. 
Nowadays, the market offers many devices dedicated for revascularization of SFA CTO - non-imaging-guides catheters such as Crosser, TruePath, Frontrunner, but only one of these has built-in optical coherence tomography (OCT) - the Ocelot catheter [5-7]. The OCT provides spatial resolution that is ten times greater compared to intravascular ultrasound (IVUS) currently applied in atherectomy devices [8]. Real time high-resolution visualization may facilitate navigation through long occlusions allowing recanalization in the true lumen, thus avoiding subintimal recanalization, and intraluminal crossing may have the benefit of reducing inflammatory reaction. The OCT give a 3-dimensional rendering of the artery wall and the operator is able to use this information for stent apposition, vulnerable plaque location and target vessel for endovascular treatment. Thanks to intraluminal recanalization physicians may have a wide array of endovascular strategies, including angioplasty, stenting and atherectomy.

\section{Aim}

Due to an increasing number of older, cardiologically high-risk patients as a result of comorbidities and the patients' demand for less invasive procedures and faster recuperation we sought to evaluate early and long-term follow-up after first-in-man (FIM) revascularization of long SFA occlusion with the OCT Guided Ocelot Catheter.

\section{Material and methods}

The paper concerns a prospective single-center nonrandomized first-in-man registry of patients with CTO SFA and severe claudication treated with the Ocelot Catheter (before receiving the CE mark). The purpose of this study was to evaluate short- and long-term outcomes after CTO SFA revascularization using the Ocelot catheter. The periprocedural outcomes as well as vessel patency at 6 months and 5 years of the intervention were evaluated using angiography and Doppler ultrasound (according to study protocol - AVI-OCT-10003 v.0; PB/ WKWM/21/2010), respectively.

\section{Patient eligibility and study requirements}

Patients were considered eligible for the study if they were diagnosed with chronic limb ischemia graded as Rutherford 3; target vessel diameter $>5 \mathrm{~mm}$ and target lesion total occlusion. The exclusion criterion was target limb necrosis, i.e., stage 6 according to the Rutherford classification. All patients were maintained on aspirin (75$150 \mathrm{mg}$ ), clopidogrel (75 mg) and statin 2 days prior the intervention. Other medications were prescribed at the physician's discretion. An intra-arterial bolus of $5000 \mathrm{IU}$ heparin was administered during the interventional procedure.

\section{Definitions}

The CTO was defined as complete occlusion of the artery. Vessel patency was defined as diameter stenosis $\leq 50 \%$. Inability to cross the lesion, perforation, no reflow and bailout stenting were evaluated as periprocedural outcomes. Serious adverse events comprised cardiovascular death, myocardial infarction, stroke, non-target lower limb vessel revascularization and amputation.

\section{Description of Atherectomy System}

Ocelot is a unique CTO-dedicated atherectomy system with built-in OCT for intravascular imaging. This catheter utilizes spiral-fluted wedges to corkscrew the CTO cap while real time OCT provides visualizations to facilitate intravascular navigation. OCT is located at the
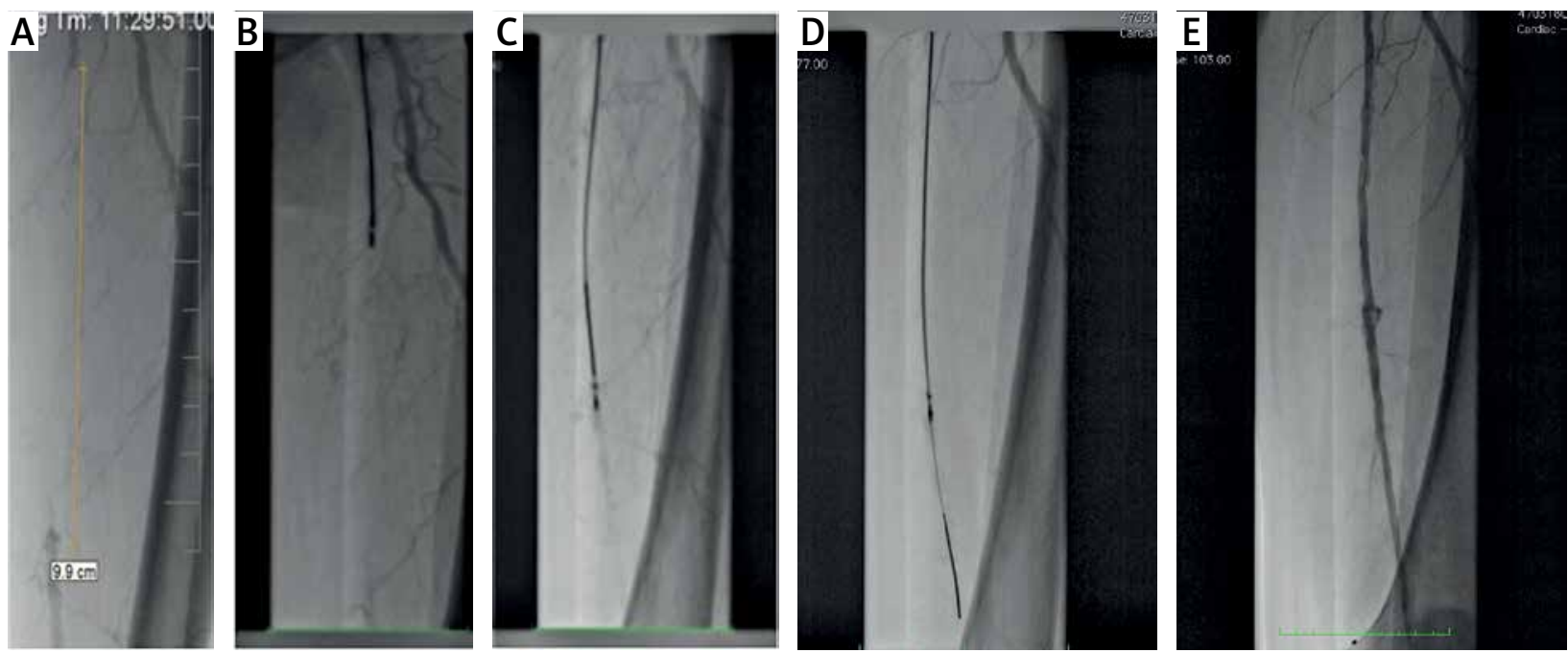

Figure 1. A - SFA CTO, B - OCELOT catheter in working position, C - OCELOT catheter in working position. Crossing medium SFA the lesion. $\mathbf{D}$ - Inserting 0.35 guidewire to distal part SFA. E - Final effect after balloon angioplasty (5 $\mathrm{mm}$ of diameter) 
Table I. Baseline demographics, comorbidities and lesion characteristics

\begin{tabular}{lc} 
Parameter & Value \\
\hline Male: female, $n$ (\%) & $9: 1(90)$ \\
\hline Age [years]: & 1 \\
\hline $50-60$ & 7 \\
\hline $60-70$ & 2 \\
\hline $70-80$ & $5(50)$ \\
\hline Comorbidities, $n$ (\%): & $9(90)$ \\
\hline Diabetes & $6(60)$ \\
\hline Hypertension & $7(70)$ \\
\hline Hyperlipidemia & $4(40)$ \\
\hline Coronary artery disease & 10 \\
\hline Post-PCI CAD & $7.45 \pm 3.25$ \\
\hline Lesion characteristics $(n=10):$ & 10 \\
\hline Location: SFA & 5.3 \\
\hline Length [cm] & $10 / 0$ \\
\hline Total occlusion & $0 / 9 / 1$ \\
\hline Reference diameter [mm] & \\
\hline Calcification: none/moderate/severe & \\
\hline
\end{tabular}

tip of the catheter allowing high-precision navigation without contrast use. Ocelot has a length of $110 \mathrm{~cm}$ and a crossing profile of $2 \mathrm{~mm}$, and is compatible with $6 \mathrm{Fr}$ sheaths and 0.014-inch guidewires (Figure $1 \mathrm{~A}$ ). The second part of the Ocelot system is a Lightbox console, which transmits a laser to the catheter through an optical fiber. The Lightbox creates an image, which can be presented in 2 modes: 1 - sector, 2 - waterfall view.

\section{Statistical analysis}

Data are reported as means \pm SD for continuous variables and percent and absolute number for categorical variables.

\section{Results}

\section{Patient characteristics and procedure}

Baseline demographic and clinical characteristics of 10 patients are shown in Table I. The cardiovascular risk factors were prevalent. One patient had a history of myocardial infarction. A history of stroke was present in one patient with minor disability. Mean SFA occlusion (measured in QVA) length was $74.5 \pm 32.5 \mathrm{~mm}$.

Contralateral access was used in $50 \%$ of the patients due to iliac and common femoral artery anatomy. The success rate was $90 \%$. In one patient the catheter could not cross the СTO due to massive calcification. Balloon angioplasty was performed in all cases; bailout stenting for vessel dissection was performed in 4 out of 9 patients (Figures $1 \mathrm{~B}-\mathrm{E}$ ). In all cases Astron Pulsar stents were used. No protection devices were used. Artery perforation and no-reflow syndrome did not occur. No patients developed access-site complications. The mean duration of the procedure and contrast agent volume were 100 $\pm 48 \mathrm{~min}$ and $433 \pm 139 \mathrm{ml}$, respectively. In the authors' opinion this fact is strongly connected with the type of this study, i.e. first in man, and is not representative for current Ocelot procedures. During the hospital stay, particular attention was paid to hydration of patients before and after the procedure. In the group of patients, no nephropathy requiring dialysis was observed during both 30-day and 5-year follow-up.

\section{Outcomes and follow-up}

\section{0-day outcomes}

All 9 patients after successful procedure were claudication-free, i.e., asymptomatic (Rutherford 0). In all patients color duplex scan was performed, without symptoms of restenosis in examinations. The patient after a failed procedure was put on medical treatment (ASA, cilostazol, statin); the claudication distance was approximately $100 \mathrm{~m}$. All 9 patients were maintained on medical treatment - ASA $75 \mathrm{mg}$, clopidogrel $75 \mathrm{mg}$, statin $40 \mathrm{mg}$.

\section{6-month angiography follow-up}

Angiographic follow-up (according to the study protocol) performed at 6 months of the procedure revealed $89 \%$ patency of the target vessel in 8 out of 9 patients. Follow-up angiography revealed significant although non-occlusive restenosis and re-CTO of the target lesion in SFA in three and one patient, respectively. In all patients with restenotic lesions the primary treatment included CTO crossing and balloon angioplasty without stent placement. At 6 months, claudication distance decreased below $100 \mathrm{~m}$ and repeat balloon angioplasty was therefore performed. Percutaneous revascularization was also attempted in the re-CTO case but proved unsuccessful. Consequently, common and deep femoral artery endarterectomy with patch plasty was performed.

\section{5-year Doppler ultrasound follow-up}

Doppler ultrasound performed at 1, 2 and 5 years of the primary intervention did not reveal important target vessel restenosis above $50 \%$ and peak systolic velocity $(P S V)>1.5 \mathrm{~m} / \mathrm{s}$. The primary and primary-assisted patency was $89 \%$. During a 5 -year follow-up, four peripheral percutaneous interventions and one femoro-popliteal by- 
pass surgery were performed in non-target limbs. There were no cardiovascular deaths, myocardial infarction or stroke and no amputation was required. Throughout the follow-up all patients were maintained on medical treatment - ASA $75 \mathrm{mg}$, clopidogrel $75 \mathrm{mg}$, statin $40 \mathrm{mg}$. The long-term double antiplatelet treatment in the $\mathrm{H}_{2}$ blocker cover was conducted in accordance with the authors' belief that the prevention of cardiovascular events was more effective.

The target limb of eight patients remained asymptomatic (Rutherford 0) from month 6 and throughout year 5 of the primary intervention. Claudication distance in the patient after patch plasty repair remained unchanged (i.e., approximately $100 \mathrm{~m}$ ).

The patient in whom CTO crossing failed due to massive calcification underwent femoro-popliteal bypass surgery 2 years after the primary intervention due to shortening of claudication distance below $50 \mathrm{~m}$.

\section{Discussion}

The CTO SFA recanalizations are mostly performed using the subintimal approach which undoubtedly results from lesion type and operator experience. New tools specially designed for intraluminal interventions allow efficient and safe treatment of long and heavily calcified CTOs that would previously be qualified (TASC II) for surgical repair.

The current study is a direct observational first-inman registry of patients with CTO revascularized with an optical coherence tomography (OCT) Guided Ocelot Catheter (Avinger). According to available research, the registry for the first time describes very long-term follow-up after OCT guided CTO of SFA revascularization. Moreover, this study was designed to obtain the CE (Conformite Européenne) mark for the OCT Guided Ocelot Catheter (Avinger). All subjects included in the study were high cardiovascular risk, symptomatic patients with long SFA CTO. Those factors determined that they were high-risk for open bypass surgery. Nonetheless, long SFA CTO was actually classified as TASC D, which should be treated with the open surgical procedure rather than endovascular [9]. The OCT Guided Ocelot Catheter is designed for chronic total SFA occlusion revascularization and $10 \mu \mathrm{m}$ image resolution allows differentiation between various healthy arterial structures including media and adventitia, and diseased arterial walls including atherosclerotic plaque [10]. The high resolution visual guidance would minimize media injury which could yield better long-term outcomes and patency [11].

The first large trial designed to assess safety of the Ocelot Catheter was the single arm prospective multicenter CONNECT study which included 84 patients with femoropopliteal CTO. Efficiency defined as successful CTO crossing with the device was achieved in $89 \%$ of included patients. Perforations were reported in $5 \%$ of cases, resolved with prolonged balloon occlusion. The clinically significant perforations were not observed [12]. In the CONNECT II study outcomes were very similar with $72 \%$ successful crossing with Ocelot alone, whereas $97 \%$ were successful when combined with assist or re-entry devices [13]. In the prospective, single-arm, multicenter Vision study of the Ocelot Catheter combined with adjunctive therapy such as stent implantation or used alone outcomes were encouraging. One hundred fifty-eight patients were enrolled, and 198 lesions were treated. The safety profile was satisfactory with no clinically significant perforations, $0.5 \%$ dissections and $2 \%$ embolic events. The clinically driven repeat target lesion revascularization was $6.4 \%$ in the 6 -month follow-up [14]. Stavroulakis et al. published long-term outcomes of endovascular revascularization with the Ocelot Catheter combined with local antimitotic drug delivery by drug-eluting balloons (DEB). The results are encouraging; in the 33 patients and 37 lesions only $5 \%$ had TLR in 12 months and low periprocedural complications [15]. Moreover, OCT Guided Ocelot Catheter is suitable even for very long, calcified CTOs involving the whole SFA [16].

Those studies confirm our observations on safety and feasibility outcomes. Moreover, the long-term follow-ups are also encouraging and comparable to our study. When comparing re-entry devices, the safety and feasibility are similar if they are used in short CTO SFA. When performed in longer CTO the efficiency ratio is more encouraging when orbital atherectomy with OCT is used [17].

Nowadays, when local drug delivery is more and more efficient this technology may by combined with orbital atherectomy in PAD revascularization. Early reports on the combination of plaque modification with atherectomy and subsequent drug-eluting balloons (DEB) seem to be promising $[18,19]$. Novel technologies, including local drug delivery nano-technology, may soon become available for the follow-up treatment of plaque modifications after atherectomy.

\section{Study limitations}

The main drawbacks of this analysis are those inherent to any single-center observational study [20]. The first-in-man nature of this study could have a significant influence on long-term outcomes due to the learning curve. This is a hypothesis-generating study rather than conclusive.

\section{Conclusions}

This first-in-man study with long-term follow-up showed that the OCT Guided Ocelot Catheter has a high success rate of CTO recanalization and very promising long-term outcomes. Nevertheless, the long-term outcomes should be evaluated in comparison with re-entry 
devices and drug technology in a prospective randomized trial.

\section{Conflict of interest}

The authors declare no conflict of interest.

\section{References}

1. Norgren L, Hiatt WR, Dormandy JA, et al. Inter-Society Consensus for the Management of Peripheral Arterial Disease (TASC II). J Vasc Surg 2007; 45 Suppl S: S5-67.

2. Tai Z. Transcollateral approach for percutaneous revascularization of complex superficial femoral artery chronic total occlusion. J Invasive Cardiol 2013; 25: E96-100.

3. Cawich I, Marmagkiolis K, Cilingiroglu M. Ocelot catheter for the treatment of long SFA occlusion. Catheter Cardiovasc Interv 2014; 83: 144-7.

4. Sakes A, Regar E, Dankelman J, Breedveld P. Crossing total occlusions: navigating towards recanalization. Cardiovasc Eng Technol 2016; 7: 103-17.

5. Liang GZ, Zhang FX. Novel devices and specialized techniques in recanalization of peripheral artery chronic total occlusions (CTOs): a literature review. Int J Cardiol 2013; 165: 423-9.

6. Kougias P. Innovative technologies for SFA management. J Cardiovasc Surg 2013; 54: 431-2.

7. Bhatt $\mathrm{H}$, Janzer $\mathrm{S}$, George JC. Crossing techniques and devices in femoropopliteal chronic total occlusion intervention. Cardiovasc Revasc Med 2017; 18: 623-31.

8. Bezerra HG, Costa MA, Guagliumi G, et al. Intracoronary optical coherence tomography: a comprehensive review clinical and research applications. JACC Cardiovasc Interv 2009; 2: 1035-46.

9. Hardman RL, Jazaeri O, Yi J, et al. Overview of classification systems in peripheral artery disease. Semin Intervent Radiol 2014; 31: 378-88.

10. Sewall LE. Treatment of chronic total occlusions using the avinger ocelot crossing catheter. Semin Intervent Radiol 2015; 32: 370-3.

11. Bunt TJ, Manship L, Moore W. latrogenic vascular injury during peripheral revascularization. J Vasc Surg 1985; 2: 491-8.

12. Pigott JP, Raja ML, Davis T; Connect Trial I. A multicenter experience evaluating chronic total occlusion crossing with the Wildcat catheter (the CONNECT study). J Vasc Surg 2012; 56: 1615-21.

13. Selmon MR, Schwindt AG, Cawich IM, et al. Final results of the Chronic Total Occlusion Crossing With the Ocelot System II (CONNECT II) study. J Endovasc Ther 2013; 20: 770-81.

14. Schwindt AG, Bennett JG Jr, Crowder WH, et al. Lower extremity revascularization using optical coherence tomography-guided directional atherectomy: final results of the eValuatlon of the pantheriS optlcal cOherence tomography imagiNg atherectomy system for use in the peripheral vasculature (VISION) Study. J Endovasc Ther 2017; 24: 355-66.

15. Stavroulakis K, Bisdas T, Torsello G, et al. Optical coherence tomography guided directional atherectomy with antirestenotic therapy for femoropopliteal arterial disease. J Cardiovasc Surg 2019; 60: 191-7.

16. Cawich I, Marmagkiolis K, Cilingiroglu M. Ocelot catheter for the treatment of long SFA occlusion. Catheter Cardiovasc Interv 2014; 83: 144-7.

17. Ko YG. SFA Intervention: Intraluminal or Subintimal? Korean Circ J 2018; 48: 685-91.
18. Stavroulakis K, Bisdas T, Torsello G, et al. Combined directional atherectomy and drug-eluting balloon angioplasty for isolated popliteal artery lesions in patients with peripheral artery disease. J Endovasc Ther 2015; 22: 847-52.

19. Tellez A, Dattilo R, Mustapha JA, et al. Biological effect of orbital atherectomy and adjunctive paclitaxel-coated balloon therapy on vascular healing and drug retention: early experimental insights into the familial hypercholesterolaemic swine model of femoral artery stenosis. Eurointervention 2014; 10: 1002-8.

20. Biondi-Zoccai GG, Agostoni P, Abbate A. Parallel hierarchy of scientific studies in cardiovascular medicine. Ital Heart J 2003; 4: 819-20. 